\title{
Patient centric formulations for paediatrics and geriatrics: Similarities and
}

\section{differences}

Sara M Hanning ${ }^{\mathrm{a}^{*}}$, Felipe L Lopez ${ }^{\mathrm{a}}$, Ian C K Wong ${ }^{\mathrm{a}}$, Terry B Ernest ${ }^{\mathrm{b}}$, Catherine

$$
\text { Tuleu }{ }^{\mathrm{a}}, \text { Mine Orlu Gul }{ }^{\mathrm{a}}
$$

5

a. UCL School of Pharmacy, 29-39 Brunswick Square, London WC1N 1AX, United Kingdom

b. GlaxoSmithKline Pharmaceuticals, Harlow, Essex CM19 5AW, United Kingdom

\section{*Corresponding author:}

Sara Hanning

Department of Pharmaceutics

UCL School of Pharmacy

29-39 Brunswick Square

15

London, WC1N 1AX

United Kingdom

Phone

00442077535968

Email s.hanning@ucl.ac.uk

20 Abstract

Paediatrics and geriatrics both represent highly heterogenous populations and require special consideration when developing appropriate dosage forms. This paper discusses similarities, differences and considerations with respect to the development of appropriate medicine formulations for paediatrics and geriatrics. Arguably the

25 most significant compliance challenge in older people is polypharmacy, whereas for children the largest barrier is taste. Pharmaceutical technology has progressed rapidly and technologies including FDCs, multi-particulates and orodispersible dosage forms provide unprecedented opportunities to develop novel and appropriate formulations for both old and new drugs. However, it is important for the formulation scientists to work closely with patients, carers and clinicians to develop such formulations for both the paediatric and geriatric population.

\section{Key words}

Paediatric, geriatric, medicine, excipients, dosage form, patient-centric 
35 Paediatric and geriatric patients do not fall into the category of 'standard patient' due to altered pharmacokinetics, different acceptable dosage forms, formulation composition and route of administration. In the paediatric population, there are distinct physiological differences between neonates, infants, children and adolescents. However, relating this information to adult data when determining an appropriate 40 dosing regimen is complicated (Bartelink et al., 2006). In neonates and infants, immaturity of enzymes, volume of distribution and clearance may result in differences in pharmacokinetics. In older people, these differences cannot be defined by age alone. Pharmacokinetics are strongly influenced by morbidity, co-morbidity, multiple drug use or reduced organ function. The ICH Harmonised Tripartite Guideline:

45 Studies in Support of Special Populations: Geriatrics E7 highlights the need to conduct pharmacokinetic studies in healthy geriatric subjects or volunteers with the disease to be treated by the drug of interest. It is not uncommon for clinical trials to exclude older patients, for reasons such as concomitant conditions, polypharmacy or frailty, yet this data is essential to maintain safety and optimise medication for the older population (Ford, 2000; Mangoni and Jackson, 2004). If age-related differences are found that could be of medical importance, a larger, multiple-dose PK study may be necessary to permit statistical comparisons between different patient cohorts at steady state (International Conference on Harmonisation, 1993). Similarly, the Paediatric Regulation was introduced in 2007 to ensure that medicines for use in 55 children are of high quality, ethically researched and appropriately authorised.

In both paediatric and geriatric population groups, challenges exist in the development of formulations that will offer a predictable and safe drug release in the patient, whilst also being presented in an acceptable dosage form to ensure safety and compliance.

60 Manufacturing complexity and cost are also important considerations. From an industry perspective, the paediatric population represent a small market, with many illnesses short term. Adopting a patient centric approach for such a small target group can be difficult financially, requiring significant labour and resources. The geriatric population, on the other hand, are a wider group with a broad range of therapeutic,

65 hence pharmaceutical, needs. By considering the similarities between the paediatric and geriatric population, labour and resource costs may be minimised whilst maintaining this patient focus. This paper outlines some of the paediatric and geriatric formulation needs from a patient centric perspective, with a focus on novel 
oral systems such as fixed-dose combinations, multi-particulates and orodispersible dosage forms. Patient centric formulation development refers to considering the end user from the beginning of the formulation process and right through the development to an end product.

\section{Excipient and other formulation issues}

75 Excipients of medications that may be acceptable in adult formulations may not be suitable for special populations such as paediatrics and geriatrics. For example, high sodium intake disturbs electrolyte balance, causing water retention and increasing the risk of cardiovascular conditions including stroke, hypertension and heart failure, particularly in older adults (George et al., 2013). Despite this, a recent review of cardiovascular formulations listed in the British National Formulary (BNF) found instances where effervescent, dispersible and soluble tablets prescribed for cardiovascular disorders contained sodium levels higher than the recommended daily intake of sodium in adults (2.4g or 104mmol) (Hanning et al., 2015; Joint Formulary Committee, 2013). In addition, only $40 \%$ of medicines listed in the BNF for

85 cardiovascular disorders specified dose recommendations that could be adjusted for older patients, taking into consideration factors such as comorbidity, polypharmacy and vulnerability to adverse effects (Hanning et al., 2015).

For children the situation is even more critical, as the vast majority of medicines prescribed for children with cardiovascular problems are unlicensed and often manipulated at the point of administration or only available as extemporaneous formulations (Standing and Tuleu, 2005). Implications of this include dosing accuracy, unknown bioavailability of extemporaneously prepared formulations, use of excipients that may be toxic and a lack of access to modified release preparations for children. Although the introduction of the European Union regulation on medicinal products for paediatric use in 2007 has endeavoured to improve rational, evidencebased prescribing and age-appropriate formulations for children, a significant number of products still lack paediatric information (Breitkreutz; Frattarelli et al., 2014; Sachs et al., 2012).

Oral drug delivery is the most popular route of medicine administration. Advantages include ease of ingestion, avoidance of administration discomfort/pain, low 
manufacture cost, versatility and expected better patient compliance (Sastry et al., 2000). Many individuals find it difficult to swallow tablets and hard gelatin capsules and this difficulty is especially prevalent in paediatric and geriatric patients (Lindgren and Janzon, 1991; Patel et al., 2015). Co-administration with food is often recommended to ease ingestion of medication, although this practice might have an impact on the oral bioavailability of the drug. Depending on the active moiety and the type of food this can result in an increased or decreased exposure (Martinez and

110 Amidon, 2002). Therefore, recommendations need to be made in a case-by-case basis. Critically, food preferences may vary between paediatric and geriatric individuals, so a variety of food types need to be considered. Not only food, but also oral vehicles (syrups and gels) and thickening agents (which can be added to a drink to increase its consistency) have been investigated and proposed. These administration aids could be

115 supplied along with the drug product, could be commercially available as a separate product, or could be extemporaneously prepared in community pharmacies as required (Kluk and Sznitowska, 2014). Caution must be taken with recommending these products until sufficient scientific evidence with regards to the safety of this practice is generated. In fact, preliminary data suggest that thickening agents could

120 hinder release of drugs from crushed tablets (Manrique et al., 2014). Further research in this topic is required to enable safe administration of medication with food and thickening agents.

\section{Fixed-dose combinations}

125 Fixed-dose combinations (FDCs) are a way of administering multiple medications in a single dosage form. Their primary advantage is to reduce complexity of therapy and improve medication compliance by reducing pill burden in patients with comorbidities. Therefore, FDCs address two key determinants of poor medication compliance - polypharmacy and the complexity of treatment regimen. FDCs have

130 been shown to decrease the risk of medication non-compliance in patients with chronic conditions (Bangalore et al., 2007). In addition, the combination of drugs with different mechanism of action can achieve greater efficacy (synergistic effect) with a lower occurrence of adverse events compared to increasing the dose of the monotherapy (Garber et al., 2002; Panaccione et al., 2014). Other advantages include 135 the simplification of drug handling and lower packing and shipping costs. FDCs are primarily advantageous for geriatric patients with polypharmacy, however, can also 
be helpful for paediatrics in conditions requiring combined medication, such as tuberculosis and HIV. Although some commercial FDC preparations exist, such as Rifater $^{\circledR}$ and Rifanah ${ }^{\circledR}$ (Sanofi-Aventis) for the treatment of tuberculosis, these are not

140 licenced for use in children. However, these preparations could be considered in older children provided that the dose of each drug is appropriate given the weight of the child (BMJ Group, 2011).

FDCs also have some potential limitations. FDCs restrict individual dose titration of each active ingredient which, indeed, discourages adjustment of doses to the individual patient's need (Blomberg et al., 2001; World Health Organisation, 2003). This is of critical importance when the combined drugs exhibit different pharmacokinetics and/or pharmacodynamics. Unless each active ingredient is available as a separate drug product, FDCs encourage polypharmacy irrespective of

150 the appropriateness of drug combination for a particular patient (World Health Organisation, 2003). The incorporation of various drugs in single dosage forms pose unprecedented technical challenges which arise from incompatibilities of the combined drugs (Singh et al., 2001). Furthermore, the final dosage form may become significantly larger, obstructing oral administration (Desai et al., 2013). This is of

155 particular importance if an individual suffers from dysphagia or struggles to swallow tablets, which are common features in the geriatric and paediatric population. Some of these challenges might be overcome via the preparation of multi-particulate formulations or oral fast dissolving dosage forms.

\section{Multi-particulate formulations}

Compared to single dose units, which usually take the form of a tablet, multiparticulate formulations are smaller, multiple unit systems of mini-tablets or pellets that are either filled into capsules or compressed into tablets that disintegrate into the original pellet size on administration (Newton, 2010). In some cases, the dose may be 165 adapted to meet patient requirements, for example the administration of a quantity of pellets based on body weight. The utilisation of specialised counting and dosing devices may be necessary in these instances (Wening and Breitkreutz, 2011) and new research is on-going in this area. 
170 Commercial examples of multiparticulate formulations include Depakote ${ }^{\circledR}$ capsules (divalproex sodium) and Creon ${ }^{\circledR}$ capsules (pankrelipase), whereby the capsules can be swallowed whole, or if swallowing is an issue the capsule contents may be sprinkled onto soft food. As discussed previously, the type of food that is used as the vehicle in these instances is important, although often little instruction is given.

175

Multi-particulate formulations are a good choice for the development of FDC products since individual dosage units containing different entities can be combined in the final dosage form (e.g. filled into capsules). This approach clearly presents fewer limitations from a pharmaceutical development perspective than the combination of drugs in the same dosage unit, particularly in the case of drugs with physical or chemical incompatibilities (Desai et al., 2013). Thus, multi-particulate formulations offer great design flexibility by combining particles with different drugs and/or with different release profiles. This type of formulation is also a great candidate for the preparation of controlled release products with minimal risk of dose dumping. Due to their reduced size, multi-particulates are expected to exhibit a shorter and more reproducible gastric emptying than single-unit dosage forms, which is desirable in the design of controlled release products. However, evidence in this area is limited in the young and the old, as studies have focused in the adult population only (Newton, 2010; Varum et al., 2010). Paediatric and, in particular, 190 geriatric patients with chronic conditions may benefit from controlled release products to reduce the frequency of administration and ultimately the pill burden.

Many barriers and unknowns arise at the point of administration of pellets and minitablets. The maximum number of dosage units that can be administered in a single 195 dose has not yet been investigated for any targeted patient group. This is important as it defines the maximum dose that can be delivered, which could hinder the preparation of FDC if the dose required exceeds the maximum delivery dose.

In spite of the acclaimed advantages of this type of formulation, the number of products in the market is still limited. The development of multi-particulate systems may require advanced pharmaceutical technology, multiple step processes and diligent control of processing variables. This can entail a time-consuming and costly production with respect to conventional solid dosage forms (Roy and Shahiwala, 
2009), although in practice multi-particulates could be manufactured in the same way as conventional tablets but down to $1 \mathrm{~mm}$ diameter (Tissen et al., 2011), using an established and well-controlled process. It is important that the combination of multiparticulate technology with the selected drug substance and packaging system is prosperous to achieve patient acceptance and smooth the path for other medicines to take the form of multi-particulate products. Oral fast-dissolving dosage forms are a more established platform that have already come a long way, with many patients already benefiting from this novel approach to drug delivery in various therapeutics areas.

\section{Orodispersible dosage forms}

215 Orodispersible dosage forms are those that disintegrate or dissolve rapidly in the oral cavity, resulting in a solution or suspension without the need for water. Examples of commercially manufactured orodispersible dosage forms are highlighted in Table 1. In terms of specific use for paediatric and geriatric population groups, their primary advantage is their ability to be administered to those with difficulties swallowing solid dosage forms (Sastry et al., 2000). However, disadvantages include limited drug loading and the requirement for taste masking.

Orodispersible tablets (ODTs) and oral lyophilisates dominate the market of oral fastdissolving dosage forms (Slavkova and Breitkreutz, 2015). Similar in appearance to conventional tablets, these solid formulations disintegrate quickly in the oral cavity thanks to a rational selection of excipients (e.g. superdisintegrants) and/or manufacturing processes which confer higher tablet porosity (Al-khattawi and Mohammed, 2014; Badgujar and Mundada, 2011). Orodispersible films (ODFs) are thin strips of film that undergo rapid disintegration in the oral cavity when placed on the tongue (Hoffmann and Breitenbach, 2011). Alternative fast-dissolving dosage forms are being introduced including orodispersible granules and orally disintegrating mini-tablets (Krause et al., 2009; Stoltenberg and Breitkreutz, 2011).

Table 1 Examples of marketed orodispersible tablets, oral lyophilisates and

235 orodispersible films. Adapted from Slavkova and Breitkreutz (2015).

Dosage form Examples of marketed products




\begin{tabular}{ll}
\hline Orodispersible tablets & $\begin{array}{l}\text { Prevacid solutab (lansoprazole), Allegra } \\
\text { (fexofenadine hydrochloride), Orapred ODT } \\
\text { (prednisolone), Parcopa (levodopa/carbidopa), } \\
\text { Zomig-ZMT (zolmitriptan) }\end{array}$ \\
\hline Oral lyophilisates & $\begin{array}{l}\text { Zofran Zydis (ondansetron), Imodium akut lingual } \\
\text { (loperamide), Zyprexa Velotab (olanzapine), } \\
\text { Clarinex Reditabs (loratadine), Zelapar (selegiline), } \\
\text { Maxalt-lingua (Rizatriptan benzoate) }\end{array}$ \\
\hline Orodispersible films & Setofilm (ondansetron), Zuplenz (ondansetron), \\
& Risperidone Hexal SF (risperidone), Donezepil-HCl \\
& Hexal SF (Donezepil hydrochloride) \\
\hline
\end{tabular}

A fundamental gap in the development of fast-dissolving dosage forms is the lack of officially recognised characterisation methods. According to the European Pharmacopoeia 7.4, orodispersible preparations should be produced in a way that they 240 possess suitable mechanical strength to withstand handling without being damaged, but the only specific test mentioned is dissolution. Despite this, there is no clear indication of how long an orodispersible preparation should take to disintegrate and there is no clearly defined endpoint for disintegration in the European Pharmacopoeia. This is particularly important as the disintegration time is a vital property that affects 245 drug administration and drug release (Pein et al., 2014). Compendial disintegration testing has shown very poor correlation with in vivo disintegration time in the mouth, while novel testing techniques can attain much better correlation (Brniak et al., 2012; Hoashi et al., 2013; Szakonyi and Zelkó, 2013). In addition to fast disintegration other ideal properties include flexibility (in the case of films), physical stability, good 250 handling and suitable mechanical strength (Visser et al., 2015).

Palatability and in particular taste are key attributes of oral fast-dissolving dosage forms as the formulation is intended to disintegrate in the oral cavity. The utilisation of taste-masking technologies in combination with oral fast-dissolving dosage forms 255 is often required (Douroumis, 2011). Several techniques might be considered, either alone or in combination, to attain taste masking. The addition of sweeteners and flavouring agents are often the first approach investigated since special 
manufacturing technologies or equipment are not required and the pharmacokinetic properties of the drug product are not likely to be affected (Walsh et al., 2014). The use of complexation (e.g. ion exchange resins) or coating (e.g. sugar, polymeric or lipidic coating) to apply a molecular or physical barrier between drug and palate is considered to be more effective than the sole addition of sweeteners and flavours; however, this approach could have an impact on the bioavailability of the product and is also more technically challenging (Walsh et al., 2014). Selection of the most appropriate taste-masking technique needs to be rationalised based on the physicochemical and organoleptic properties of the drug.

The evaluation of taste and overall palatability is becoming common practice. Taste assessment can be performed by means of dissolution testing in bio-relevant

270 conditions (Tan et al., 2013), in vitro techniques such as electronic tongues (Preis et al., 2012), in vivo animal models (Noorjahan et al., 2014), or with a panel of human volunteers (Pein et al., 2014). Studies using human taste panels are typically conducted in adults, which can be problematic when the target population is paediatrics and geriatrics, who have different taste sensations (Krause and

275 Breitkreutz, 2008). Appropriate palatability is particularly important in the case of medicines for children. The current European guidelines urge the assurance of appropriate palatability in paediatric products (European Medicines Agency, 2013).

\section{The future direction of pharmaceutical development of medicines for paediatric} and geriatric use

Both geriatric and paediatric patients, particularly of extreme age, sometimes find it difficult to swallow; therefore, flexible dosing and appropriate strengths of formulations are needed. In terms of difference in disease incidence and distribution in neurological disorders, pharmaceutical research in older patients should focus on

285 neurodegenerative diseases and disorder due to neuro-insult such as Parkinson's disease, Alzheimer's, stroke and epilepsy, all these illnesses still require appropriate formulation to assist the carers to administer and patient to swallow. Similarly, neurodevelopmental disorders in children also create significant challenges. Illnesses such as Autism Spectrum Disorder, cerebral palsy and epilepsy all require careful

290 formulation research to develop appropriate medicines that assist the ease of administration for the carers and also make it easy for the patient to swallow. 
Another important clinical area for formulation research is palliative care for both older people and children. As the population ages, there is clearly an increased need

295 for palliative care. Palliative care patients require medications to be administered within their homecare settings to allow them to stay in a familiar environment; however, many medicines for fast symptom relief require injection. This route could be potentially replaced by non-invasive dosage forms, such as orodispersible and oromucosal formulations and current research is working towards addressing this.

300 The above-mentioned issues are also applied to paediatric palliative care, but with the added complexity due to the small dose requirement that the appropriate formulation is very important to avoid accidental overdose.

The most appropriate dosage form and manufacturing technology need to be selected 305 considering the physicochemical properties of the drug, but also the target population. Novel technologies including inkjet and 3D printing bring unparalleled opportunities for the preparation of personalised medicines, either in industrial settings or in hospitals and community pharmacies. Investment and development of infrastructure are required for this to be feasible and adaptations of regulatory framework are

310 already underway to support this (Food and Drug Administration, 2013). In August 2015, a 3D-printed drug became the first of its type to be approved by the FDA, which reinforces promise for this direction. Spritam (levetiracetam), developed by Aprecia Pharmaceuticals (Langhorne, Pennsylvania), uses the company’s ZipDose Technology platform, which applies powder-liquid 3D printing to produce a porous

315 formulation that rapidly disintegrates with a small volume of liquid (Voelker, 2015).

Pharmaceutical technology has progressed rapidly and many of the above-mentioned technologies provide unprecedented opportunities to develop novel and appropriate formulations for both old and new drugs. However, it is important for the formulation scientists to work closely with patients, carers and clinicians to develop such formulations for both older people and children.

\section{Conclusions}

Paediatric and geriatric populations deviate from the standard patient with respect to 325 both pharmacokinetics and pharmacodynamics. These changes require dose 
adaptations and careful selection of excipients when developing a dosage form.

Although there is considerable overlap when it comes to 'ideal' formulations and dosage forms for these two population groups, there are also key differences that require consideration.. The development of novel drug delivery systems including FDCs and multi-particulates may help to address some of these problems in both population groups. In addition, regulatory expectations are being established to help facilitate the development of dosage forms that are suitable for paediatric and geriatric populations. The release of further regulatory guidance documents and academic research articles as well as success stories in the form of licensed patient centric products will drive the future of paediatric and geriatric appropriate formulations.

\section{References}

340 Al-khattawi, A., Mohammed, A.R., 2014. Challenges and emerging solutions in the development of compressed orally disintegrating tablets. Expert Opin. Drug Discov. 9, 1109-1120.

Badgujar, B.P., Mundada, A.S., 2011. The technologies used for developing orally disintegrating tablets: a review. Acta Pharm. 61, 117-139.

Bangalore, S., Kamalakkannan, G., Parkar, S., Messerli, F.H., 2007. Fixed-dose combinations improve medication compliance: a meta-analysis. Am. J. Med. 120, 713-719.

Bartelink, I., Rademaker, C.A., Schobben, A.A.M., van den Anker, J., 2006. Guidelines on paediatric dosing on the basis of developmental physiology and pharmacokinetic considerations. Clin. Pharmacokinet. 45, 1077-1097.

355 Blomberg, B., Spinaci, S., Fourie, B., Laing, R., 2001. The rationale for recommending fixed-dose combination tablets for treatment of tuberculosis. Bulletin of the World Health Organization 79, 61-68.

BMJ Group, 2011. Antituberculosis drugs, British National Formulary for 360 Children. Pharmaceutical Press, London, UK.

Breitkreutz, J., 2008. European perspectives on pediatric formulations. Clin. Ther. 30, 2146-2154.

365 Brniak, W., Jachowicz, R., Krupa, A., Skorka, T., Niwinski, K., 2012. Evaluation of co-processed excipients used for direct compression of orally disintegrating tablets (ODT) using novel disintegration apparatus. Pharm. Dev. Technol. 18, 111. 

challenges, and development considerations for fixed dose combination (FDC) of oral solid dosage forms. Pharm. Dev. Technol. 18, 1265-1276.

Douroumis, D., 2011. Orally disintegrating dosage forms and taste-masking technologies; 2010. Expert opinion on drug delivery 8, 665-675.

European Medicines Agency, 2013. Medicines for older people. http://www.ema.europa.eu/ema/index.jsp?curl=pages/special topics/general/ general content 000249.jsp\&mid=WC0b01ac058004cbb9. Accessed $30 / 03 / 2014$.

Food and Drug Administration, 2013. Paving the way for personalized medicine: FDA's role in a new era of medical product development. U.S. Food and Drug Administration, Silver Spring, MD.

Ford, G.A., 2000. Pharmacodynamics, in: Crome, P., Ford, G.A. (Eds.), Drugs and the older population. Imperial College Press, London, pp. 90-101.

Frattarelli, D., Galinkin, J., Green, T., Johnson, T., Neville, K., Paul, I., Van Den Anker, J., American Academy of Pediatrics Committee on Drugs, 2014. Off-label use of drugs in children. Pediatrics 133, 563-567.

Garber, a.J., Larsen, J., Schneider, S.H., Piper, B.a., Henry, D., 2002. Simultaneous glyburide/metformin therapy is superior to component monotherapy as an

395 initial pharmacological treatment for type 2 diabetes. Diabetes Obes. Metab. 4, 201-208.

George, J., Majeed, W., Mackenzie, I.S., MacDonald, T.M., Wei, L., 2013. Association between cardiovascular events and sodium-containing effervescent, dispersible, 400 and soluble drugs: nested case-control study. BMJ 347.

Hanning, S.M., Muhamed, J., Orlu-Gul, M., 2015. Investigation into the dosage form attributes of currently UK licensed cardiovascular and Parkinson's disease drug products. Int. J. Pharm. 479, 159-162.

Hoashi, Y., Tozuka, Y., Takeuchi, H., 2013. Development of a novel and simple method to evaluate disintegration of rapidly disintegrating tablets. Chem. Pharm. Bull. 61, 962-966.

410 Hoffmann, E.M., Breitenbach, A., 2011. Advances in orodispersible films for drug delivery. Expert Opinion on Drug Delivery 8, 299-316.

International Conference on Harmonisation, 1993. Studies in support of special populations: Geriatrics E7.

415 http://www.ich.org/fileadmin/Public Web Site/ICH Products/Guidelines/Effica cy/E7/Step4/E7 Guideline.pdf. Accessed 28/09/15 
Joint Formulary Committee, 2013. British National Formulary, 66th ed. BMJ Group and Pharmaceutical Press, London.

Kluk, A., Sznitowska, M., 2014. Application properties of oral gels as media for administration of minitablets and pellets to paediatric patients. Int. J. Pharm. 460, 228-233.

425 Krause, J., Breitkreutz, J., 2008. Improving drug delivery in paediatric medicine. Pharm. Med. 22, 41-50.

Krause, J., Thommes, M., Breitkreutz, J., 2009. Immediate release pellets with lipid binders obtained by solvent-free cold extrusion. Eur. J. Pharm. Biopharm.

$430 \quad 71,138-144$.

Lindgren, S., Janzon, L., 1991. Prevalence of swallowing complaints and clinical findings among 50-79-year-old men and women in an urban population. Dysphagia 6, 187-192.

Mangoni, A.A., Jackson, S.H., 2004. Age-related changes in pharmacokinetics and pharmacodynamics: basic principles and practical applications. Br. J. Clin. Pharmacol. 57, 6-14.

440 Manrique, Y.J., Lee, D.J., Islam, F., Nissen, L.M., Cichero, J.a.Y., Stokes, J.R., Steadman, K.J., 2014. Crushed tablets: Does the administration of food vehicles and thickened fluids to aid medication swallowing alter drug release? J. Pharm. Pharm. Sci. 17, 207-219.

445 Martinez, M.N., Amidon, G.L., 2002. A mechanistic approach to understanding the factors affecting drug absorption: a review of fundamentals. J. Clin. Pharmacol. $42,620-643$.

Newton, J.M., 2010. Gastric emptying of multi-particulate dosage forms. Int. J. 450 Pharm. 395, 2-8.

Noorjahan, A., Amrita, B., Kavita, S., 2014. in vivo evaluation of taste masking for developed chewable and orodispersible tablets in humans and rats. Pharm. Dev. Technol. 19, 290-295.

Panaccione, R., Ghosh, S., Middleton, S., Márquez, J.R., Scott, B.B., Flint, L., van Hoogstraten, H.J.F., Chen, A.C., Zheng, H., Danese, S., Rutgeerts, P., 2014. Combination therapy with infliximab and azathioprine is superior to monotherapy with either agent in ulcerative colitis. Gastroenterology 146, 392$460 \quad 400 . \mathrm{e} 393$.

Patel, A., Jacobsen, L., Jhaveri, R., Bradford, K.K., 2015. Effectiveness of pediatric pill dwallowing interventions: A systematic review. Pediatrics 135, 883-889.

465 Pein, M., Preis, M., Eckert, C., Kiene, F.E., 2014. Taste-masking assessment of solid oral dosage forms-A critical review. Int. J. Pharm. 465, 239-254. 
Preis, M., Pein, M., Breitkreutz, J., 2012. Development of a taste-masked Ooodispersible film containing dimenhydrinate. Pharmaceutics 4, 551-562.

Roy, P., Shahiwala, A., 2009. Multiparticulate formulation approach to pulsatile drug delivery: Current perspectives. J. Control. Release 134, 74-80.

Sachs, A.N., Avant, D., Lee, C.S., Rodriguez, W., Murphy, M.D., 2012. Pediatric information in drug product labeling. JAMA 307, 1914-1915.

Sastry, S.V., Nyshadham, J.R., Fix, J.A., 2000. Recent technological advances in oral drug delivery - a review. Pharm. Sci. Technol. To. 3, 138-145.

480 Singh, S., Mariappan, T.T., Shankar, R., Sarda, N., Singh, B., 2001. A critical review of the probable reasons for the poor variable bioavailability of rifampicin from anti-tubercular fixed-dose combination (FDC) products, and the likely solutions to the problem. Int. J. Pharm. 228, 5-17.

485 Slavkova, M., Breitkreutz, J., 2015. Orodispersible drug formulations for children and elderly. Eur. J. Pharm. Sci. 75, 2-9.

Standing, J.F., Tuleu, C., 2005. Paediatric formulations-Getting to the heart of the problem. Int. J. Pharm. 300, 56-66.

Stoltenberg, I., Breitkreutz, J., 2011. Orally disintegrating mini-tablets (ODMTs)-a novel solid oral dosage form for paediatric use. Eur. J. Pharm. Biopharm. 78, 462-469.

495 Szakonyi, G., Zelkó, R., 2013. Prediction of oral disintegration time of fast disintegrating tablets using texture analyzer and computational optimization. Int. J. Pharm. 448, 346-353.

Tan, Q., Zhang, L., Liu, G., He, D., Yin, H., Wang, H., Wu, J., Liao, H., Zhang, J., 2013. Novel taste-masked orally disintegrating tablets for a highly soluble drug with an extremely bitter taste: design rationale and evaluation. Drug Dev. Ind. Pharm. 39, 1364-1371.

Tissen, C., Woertz, K., Breitkreutz, J., Kleinebudde, P., 2011. Development of minitablets with $1 \mathrm{~mm}$ and $2 \mathrm{~mm}$ diameter. Int. J. Pharm. 416, 164-170.

Varum, F.J.O., Merchant, H.a., Basit, A.W., 2010. Oral modified-release formulations in motion: The relationship between gastrointestinal transit and drug absorption. Int. J. Pharm. 395, 26-36.

Visser, J.C., Woerdenbag, H.J., Crediet, S., Gerrits, E., Lesschen, M.A., Hinrichs, W.L.J., Breitkreutz, J., Frijlink, H.W., 2015. Orodispersible films in individualized pharmacotherapy: The development of a formulation for pharmacy preparations. Int. J. Pharm. 478, 155-163. 
Voelker, R., 2015. The printed pill. JAMA 314, 1108.

Walsh, J., Cram, A., Woertz, K., Breitkreutz, J., Winzenburg, G., Turner, R., Tuleu, C., 2014. Playing hide and seek with poorly tasting paediatric medicines: Do not

520 forget the excipients. Adv. Drug Deliv. Rev. 73, 14-33.

Wening, K., Breitkreutz, J., 2011. Oral drug delivery in personalized medicine: Unmet needs and novel approaches. Int. J. Pharm. 404, 1-9.

525 World Health Organisation, 2003. Regulatory Challenges: Regulation of fixeddose combination products, WHO Drug Information, pp. 143-227. 